\title{
Atypical Mycobacterial Keratitis: A Negligent and Emerging Thread for Blindness
}

\author{
Thet Tun Aung', Roger W Beuerman 1,2,3* \\ 'Singapore Eye Research Institute, Singapore 169856 \\ ${ }^{2}$ SRP Neuroscience and Behavioral Disorders, Duke-NUS, Singapore 169857 \\ ${ }^{3}$ Department of Ophthalmology, Yong Loo Lin School of Medicine, National University of Singapore, Singapore 119228
}

Article Info

\section{Article Notes}

Received: November 28, 2017

Accepted: February 12, 2018

\section{*Correspondence:}

Dr. Roger W Beuerman, Singapore Eye Research Institute The Academia, 20 College Road, Discovery Tower Level 6, Singapore 169856; Telephone: (65) 6322-4544, E-mail: rwbeuerman@gmail.com

(C) 2018 Beuerman RW. This article is distributed under the terms of the Creative Commons Attribution 4.0 International License.

\section{Keywords}

Atypical mycobacteria

Keratitis

Biofilm

Extracellular DNA

DNase

\section{ABSTRACT}

Atypical mycobacteria are wide spread in the environment; they are now known to be a cause of a variety of infections including corneal infections especially after refractive surgery. The diagnosis and clinical management are often unsatisfactory due to misdiagnosis and requirement of prolonged combination of antibiotics. Evolving drug resistance is known to be a unique feature in managing atypical mycobacterial keratitis due to the propensity for the development of biofilms. In this article, we provide an etiology of atypical mycobacterial keratitis, mycobacterial biofilm pathogenesis, and the importance of mycobacterial biofilm matrix component (extracellular DNA) in maintaining mycobacterial biofilm matrix maintenance. Current treatment options for atypical mycobacterial keratitis are summarized and suggestions are made for the new treatment strategies targeting on the mycobacterial biofilm pathogenesis pathway.

\section{Etiology of Atypical Mycobacterial Keratitis}

Bacterial keratitis is an important cause of corneal opacification and blindness across the world, but particularly so in South East Asia ${ }^{1}$. Several types of pathogenic organisms can lead to keratitis, inflammation of the cornea of the eye with potential corneal perforation, sight-impairing fibrosis and loss of vision ${ }^{1}$. Causal organisms for keratitis include bacteria, fungus, virus, protozoa and parasites and the risk factors include ocular trauma and surgery, contact lens wear, pre-existing ocular morbidity, dry eye, lid deformity, systemic immunosuppression and prolong usage of topical steroids ${ }^{2-4}$. In recent years, there has been an emerging trend of atypical mycobacterial (AMB) corneal infections. The rising use of biomaterials, increasing number of refractive surgery procedures [LASIK (laser-assisted in situ keratomileusis), endothelial keratoplasty] and the expanded use of fluoroquinolone prophylaxis result in the rising AMB keratitis cases ${ }^{5}$. AMB are often referred to as nontuberculous mycobacteria, and these aerobic, nonmotile, non-spore forming bacilli have been documented in many reports of ophthalmic infections such as keratitis, canaliculitis, uveitis, dacryocystitis, orbital cellulitis, and endophthalmitis ${ }^{6-9}$. AMB keratitis is a leading cause of post-LASIK infections as noted by the American Society of Cataract and Refractive survey (ASCRS) Cornea Clinical Committee ${ }^{10}$. Among the different ocular morbidities caused 
by atypical mycobacteria, infectious keratitis is seen most commonly ${ }^{11-14}$. AMB keratitis accounts for 3 percent of total different bacterial keratitis cases in the United States in $2013^{15}$. There were reports suggesting that Mycobacterium fortuitum (M. fortuitum) and Mycobacterium chelonae (M. chelonae) are the most prevalent causal strains ${ }^{5,12}$ for AMB keratitis.

AMB infections are endemic in nature, widely distributed and varied in nature depending upon the environmental factors such as geographical status, climate and soil ${ }^{11}$. Mode of transmission of AMB infections is poorly understood, but they are widespread throughout the world ${ }^{16}$ and accounting for 10 percent of total mycobacterial infections other than Mycobacterium tuberculosis (M. tuberculosis) in the United States ${ }^{17}$. One of the unusual characteristics of AMB is the lack of evidence of person-to-person transmission like M. tuberculosis. The latent forms of AMB may be present in a normal person. However, AMB may turn into an active infection when a person looses their natural immunological capacity due to other predisposing factors ${ }^{18}$. It has been postulated that AMBs are opportunistic infections resulting lesions in the lowered resistance regions. Children and older adults with chronic diseases are more susceptible to AMB infections. There were reports suggesting that a history of corneal insults with a latent period was the main barrier leading to the poor management of $M$. chelonae keratitis cases $^{13,14}$. Ocular trauma, leading to the breach of the corneal epithelial barrier function, a property of innate immunity, is a common risk factor for AMB keratitis ${ }^{19}$. Immune compromised patients or patients using steroid medications are found to be prone to AMB infections as well. There was a report suggesting that steroid use could accelerate AMB keratitis ${ }^{20}$. The portal of entry for these AMB infections is direct inoculation or entry into the skin $^{21}$ or cornea ${ }^{19}$. In keratitis cases, it is possible that AMB infections are caused by either direct contact with biomaterial or dust containing airborne mycobacteria.

\section{Atypical Mycobacterial Biofilms}

Bacterial pathogens are able to form surface-attached complex multicellular biofilm communities and according to National Institute of Health, a biofilm phase is part of $80 \%$ of all bacterial infections ${ }^{22}$, including conditions such as gingivitis, infectious kidney stones, bacterial endocarditis, and inner ear infections, along with many hospitalacquired infections from catheters and ports ${ }^{23-25}$. Bacterial cells within biofilms are surrounded by extracellular polymeric substances (EPS) consisting of extracellular proteins, extracellular polysaccharides and extracellular DNA (eDNA), and have a distinct physiology compared to their free-living counterparts ${ }^{26}$. Formation of biofilms noticeably increases the bacterial cells' tolerance toward antimicrobials as well as to attack by the host immune system $^{27,28}$. Biofilm formation is responsible for a large percentage of treatment failures for infections and causing a huge economic burden for our health care system ${ }^{27}$, ${ }^{28}$. Increasing numbers of reports suggest that different infections including ocular infections involve biofilm formation as a major therapeutic burden. Recent reports suggest that Pseudomonas aeruginosa ( $P$. aeruginosa) biofilms are found on the mucosa of the lungs in cystic fibrosis $^{29}$ and the cornea ${ }^{30}$, both are epithelial surfaces. The classical stages of biofilm formation in experimental infections ( $P$. aeruginosa and atypical mycobacteria) of mouse cornea ${ }^{30}$ have provided evidence of biofilm formation using $\mathrm{EPS}^{30}$, bio reporter strains ${ }^{32}$ and eDNA ${ }^{33}$ as biofilm markers. Therefore, we suggest that bacterial biofilms play a critical role in AMB keratitis in several ways:

1. Mycobacterial biofilms serve as a reservoir that can release bacteria leading to the secondary infection.

2. Mycobacterial biofilms are resistant to conventional therapeutic options, making it difficult to eliminate the bacteria.

3. Lateral gene transfer can occur in biofilms encouraging resistance.

4. Failure of conventional antibiotics and host immune responses to eliminate the infection lead to chronic infection, which in turn produces a chronic inflammatory response and leading to tissue damage apart from damage by bacteria directly ${ }^{34}$.

\section{Importance of Extracellular DNA in Atypical Mycobacterial Biofilm}

EPS has been recognized as a primary component of biofilms. However, eDNA has now been found to be equally important. The controversial issue of the source of eDNA in bacterial biofilms is an ongoing. Cell lysis is the major mechanism providing eDNA in P. aeruginosa, Staphylococcus spp and Enterococcus spp ${ }^{35-37}$. Autolysins proteins[36], pyocyanin leading to $\mathrm{H}_{2} \mathrm{O}_{2}$ production ${ }^{38}$ are major factors in the cell lysis. However, there has been a growing interest of research regarding active eDNA export as an alternative source of eDNA. One report suggested that there was no evidence of cell lysis in Enterococcus faecalis early biofilms with an abundance of eDNA ${ }^{39}$. Secreted membrane vesicles were reported to be responsible for active export of eDNA in biofilms ${ }^{40}$. It was found that active export of eDNA in atypical mycobacteria was positively targeted by bicarbonate ${ }^{41}$. The functional importance of eDNA in the biofilm has been shown ${ }^{42}$ and there have been several reports showing the presence of eDNA in biofilms of different bacteria species ${ }^{43}$. Therefore, eDNA plays a major and universal role in the regulation of the biofilm network and integrity in most bacterial species. Horizontal gene transfer between bacteria is another factor leading 
to antibiotic resistance in the clinic which was first investigated in $1928^{44}$. Moreover, it has been discovered that the eDNA pool in a bacterial biofilm provides a rich medium for genetic transformation naturally, other than bacteriophage induced gene transfer and mobile genetic elements ${ }^{45}$. Studies suggest that eDNA can increase gene expressions which could lead to antimicrobial resistance ${ }^{46}$. There were reports shown that eDNA is a major component of the mycobacterial biofilm matrix and DNase 1 aids the efficacy of antibiotics in treating infections in in vitro ${ }^{48,49}$. Moreover, an addition of exogenous eDNA enhances the biofilm formation in in vitro, suggesting again that eDNA plays a major and integral part of mycobacterial biofilm matrix maintenance and regulation ${ }^{33}$.

\section{Clinical Features and Diagnosis}

Symptoms of AMB keratitis such as decreased vision, a variable degree of pain and photophobia are usually present in gradually increasing patterns, probably due to the spread of the infection within the cornea ${ }^{7}$. The clinical features are less severe in post-LASIK AMB keratitis than that of cases caused by trauma ${ }^{19}$. The variable clinical presentations of AMB keratitis cases, multifocal or a single lesion surrounded by multiple white satellite lesions were reported ${ }^{7}{ }^{20}$. Most AMB keratitis cases showed an intact corneal epithelium at their initial presentations ${ }^{50}$. Hypopyon is present in poorly managed AMB keratitis cases and the anterior chamber usually appears to be normal ${ }^{7}$. An unique clinical sign, 'cracked windshield' appearance', due to radiating corneal infiltrates by AMB infections has been noted (Figure-1) ${ }^{51}$. Intrastromal opacity, infectious crystalline keratopathy and minimal inflammation have been reported in some of the cases of AMB keratitis ${ }^{52}$. The clinical diagnosis of AMB keratitis is often delayed due to the slow growing nature of the organisms ${ }^{7}$. Corneal scraping from the lesions is required to identify the AMB and the gold standard of the laboratory detection is culturing on specific solid agar media such as Lowenstein-Jensen medium, MacConkey agar, Middlebrook

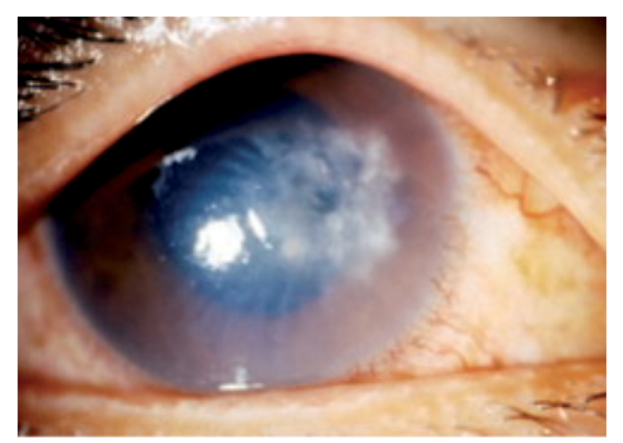

Figure-1. Slit lamp photograph showing $M$. fortuitum keratitis showing 'crack-windshield' appearance. Image is taken from Chu HS, Hu FR. Non-tuberculous mycobacterial keratitis. Clin Microbiol Infect, 2012. 19(3): 221-226 with permission ${ }^{64}$
7H10 and 7H11 media ${ }^{19}$. Molecular PCR-based technology has been developed to detect AMB infections, which are relatively faster and more specific compared to traditional microbiological methods ${ }^{53}$. Primers targeting the heat shock protein 65 (hsp65) have been utilized to detect $M$. fortuitum in postoperative endophthalmitis patients ${ }^{54}$.

\section{Therapeutic Options currently available for Atypical Mycobacterial Keratitis}

The rarity of the disease in clinical scenarios can result in complications in dealing with AMB infectious keratitis. In one report, 4 months was required for the diagnosis of a mycobacterial infection in South Flordia ${ }^{55}$. Clinical management of AMB keratitis is not efficient because of the delayed diagnosis and prolonged drug sensitivity tests due to their long incubation time to grow the bacteria in the lab. Moreover, there are several reports showing variations in MIC susceptibilities of M. fortuitum and $M$. chelonae against different antimicrobials ${ }^{56}$. According to the literature reviewed, antibiotics showed a large range of antimicrobial sensitivities, for the effective treatment of M. fortuitum and M. chelona ${ }^{56}$. Amikacin has been the gold standard treatment for AMB keratitis. However, it has been shown that amikacin is ineffective in the management of AMB keratitis and unable to act efficiently in the intact corneal epithelium ${ }^{57,58}$. Recent reports showed that fluoroquinolones are a better choice for treatment and have an excellent antimicrobial activity against AMB keratitis ${ }^{33}$, ${ }^{50}$. However, there are reports of resistance evolving for AMB against the fluoroquinolones ${ }^{59,60}$. Combination of clarithromycin and amikacin haven been used to treat AMB infections ${ }^{61,62}$. On the other hand, AMB infections in and around the eye are clinically recalcitrant to treatment, recur after cessation of therapy, and require long-term therapy to eradicate the infection. Currently there is a lack of antibiotics and settled treatment strategy that can efficiently eradicate AMB infections. The above-mentioned factors and a low index of clinical suspicion result in the inefficient management of AMB keratitis cases.

\section{Conclusion}

Although AMB keratitis cases are increasing, the understanding of the pathogenesis of AMB keratitis and effective treatment options are still limited. Studies have shown that M. fortuitum and M. chelonae readily form drug-resistant biofilms, irregular finger-like projections and microcolonies (Figure-2), suggesting a probable cause of poor treatment compliance to conventional antibiotics ${ }^{48}$. Studies have concluded that there is a significant eDNA release with $M$. fortuitum and chelonae biofilms, which protect the organisms from the antibiotics: however, adding DNase can boost the efficacy of available antibiotics ${ }^{33,48,}$ 49. There was a pioneer report to show that AMB keratitis is a biofilm mode of growth in an experimental mouse 

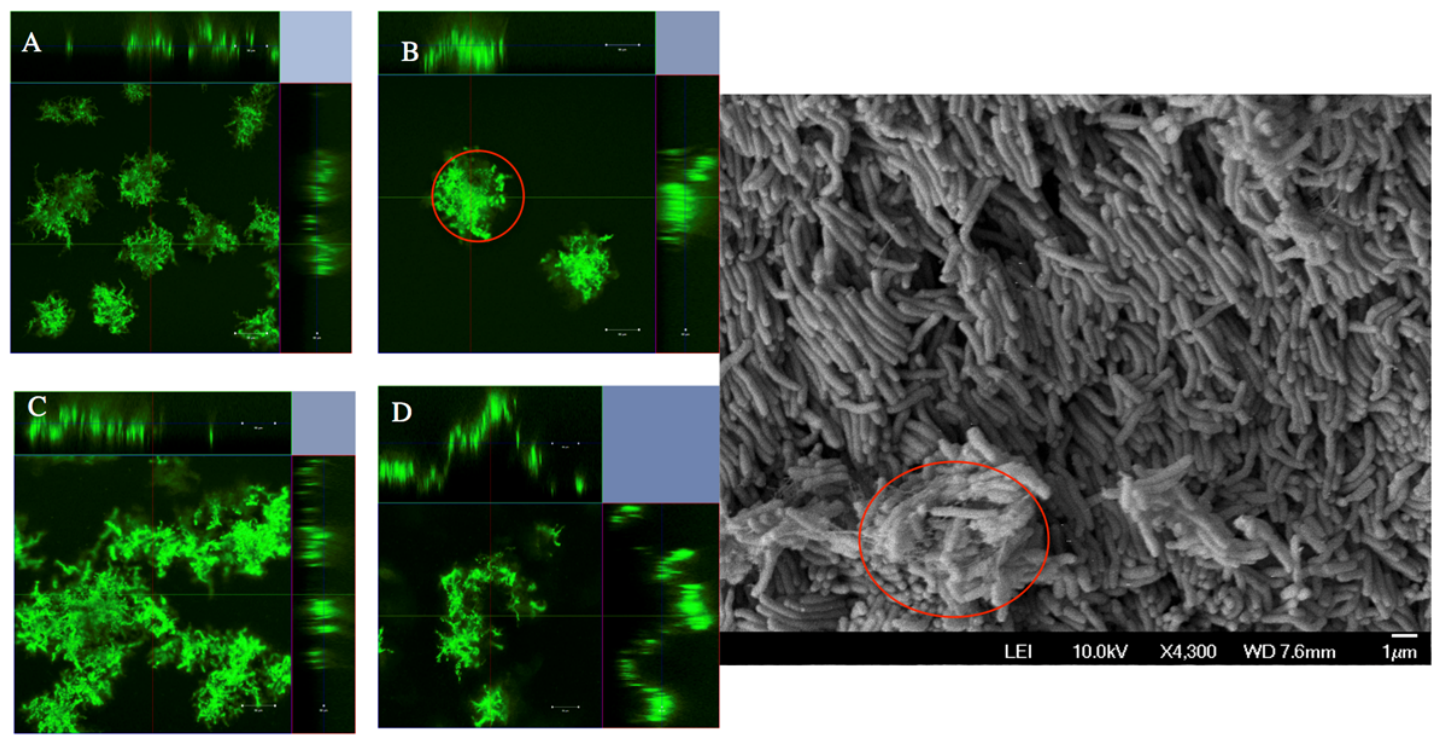

\section{A-day3 \\ B-day5 \\ C-day7 \\ D-day10}

Figure-2. M. fortuitum biofilm formation showing irregular finger-like aggregations (red circle) observed on confocal microscopy and scanning electron microscopy ${ }^{48}$

keratitis model and suggested that poor clinical outcomes of AMB infections might be due to its presence of biofilm matrix causing a barrier for antibiotic penetration and resistance ${ }^{33}$. The clinical management of AMB keratitis was often unsatisfactory due to its potential to acquire resistance because of its single agent therapy and unsettled treatment guideline ${ }^{63}$. Therefore, clinicians prescribe drug combinations in order to reduce drug resistance problems and treat AMB keratitis effectively. Since there was a report suggesting a strong synergism between DNase, aminoglycosides, and fluoroquinolones against $\mathrm{AMB}$ infections ${ }^{33}$, a consideration for a new standard treatment might be using these three different drugs in combination in any suspicious AMB infection cases. Future studies need to focus these three drugs in an optimal combination against different clinical isolates with validation in clinical trials.

\section{Acknowledgements}

The authors would like to acknowledge the support of funding from NMRC/TCR/002-SERI/2012/R1018.

\section{Conflict of Interest}

Nil

\section{References}

1. Bialasiewicz A. Triple therapeutic approach for keratitis. 1985, Infectious diseases of the eye. Buren: Aeolus Press.

2. Green M, Apel A, Stapleton F. Risk factors and causative organisms in microbial keratitis. Cornea. 2008; 27(1): p. 22-27.
3. Ahn M, Yoon KC, Ryu SK, et al, Clinical aspects and prognosis of mixed microbial (bacterial and fungal) keratitis. Cornea. 2011; 30(4): p. 409-413.

4. Bharathi M, Ramakrishnan R, Meenakshi R, et al, Analysis of the risk factors predisposing to fungal, bacterial \& Acanthamoeba keratitis in south India. The Indian journal of medical research. 2009; 130(6): p. 749.

5. Girgis DO, Karp CL, Miller D. Ocular infections caused by nontuberculous mycobacteria: update on epidemiology and management. Clinical \& experimental ophthalmology. 2012; 40(5): p. 467-475.

6. Lalitha P, Rathinam S, Srinivasan M. Ocular infections due to nontuberculous mycobacteria. Indian journal of medical microbiology. 2004; 22(4): p. 231.

7. Huang S, Soong HK, Chang JS, et al, Non-tuberculous mycobacterial keratitis: a study of 22 cases. British journal of ophthalmology. 1996; 80(11): p. 962-968.

8. Rich RM, Smiddy WE, Davis JL. Infectious scleritis after retinal surgery. American journal of ophthalmology. 2008; 145(4): p. 695-699.

9. Matieli LC, De Freitas D, Sampaio J, et al, Mycobacterium abscessus endophthalmitis: treatment dilemma and review of the literature. Retina. 2006; 26(7): p. 826-829.

10. Solomon R, Donnenfeld ED, Azar DT, et al. Infectious keratitis after laser in situ keratomileusis: results of an ASCRS survey. Journal of Cataract \& Refractive Surgery. 2003; 29(10): p. 2001-2006.

11. Turner L. Atypical mycobacterial infections in ophthalmology. Transactions of the American Ophthalmological Society. 1970; 68: p. 667.

12. Turner L, Stinson I. Mycobacterium fortuitum: as a cause of corneal ulcer. American journal of ophthalmology. 1965; 60(2): p. 329-331.

13. Broadway DC, Kerr-Muir MG, Eykyn SJ, et al. Mycobacterium chelonei keratitis: a case report and review of previously reported cases. EYELONDON-OPHTHALMOLOGICAL SOCIETY OF THE UNITED KINGDOM 
THEN ROYAL COLLEGE OF OPHTHALMOLOGISTS-. 1994; 8: p. 134134.

14. Dansby W. Epidemiological notes and reports. Mycobacterium chelonei infections following eye surgery-Texas. Morbidity and Mortality Weekly Report. 1983; 32: p. 591-600.

15. Panel, A.A.o.O.C.E.D. Preferred Practice Pattern Guidlines. Bacterial Keratitis, in American Academy of Ophthalmology. 2013: San Francisco, CA.

16. Runyon EH. Anonymous mycobacteria in pulmonary disease. The Medical clinics of North America. 1959; 43(1): p. 273-290.

17. Runyon EH. Anonymous mycobacteria in human disease. The Anonymous Mycobacteria in Human Disease. 1960; p. 3-12.

18. Youmans G. The pathogenic" atypical" mycobacteria. Annual Reviews in Microbiology. 1963; 17(1): p. 473-494.

19. Moorthy RS, Valluri S, Rao NA. Nontuberculous mycobacterial ocular and adnexal infections. Survey of ophthalmology. 2012; 57(3): p. 202235.

20. Hu FR, Huang WJ, Huang SF. Clinicopathologic study of satellite lesions in nontuberculous mycobacterial keratitis. Japanese journal of ophthalmology. 1998; 42(2): p. 115-118.

21. Linell F, Norden A. Mycobacterium balnei. A New Acid-Fast Bacillus occurring in Swimming Pools and Capable of producing Skin Lesions in Humans. Acta Tuberculosea Scandinavica. 1954.

22. Research on microbial biofilms. 2002, National Institute of Health (PA-03-047) Available online: http://grants.nih.gov/grants/guide/ pa-files/PA-03-047.html.

23. Yang L. Combating biofilms. FEMS Immunology \& Medical Microbiology. 2012; 65(2): p. 146-157.

24. Webb JS, Givskov M, Kjelleberg S. Bacterial biofilms: prokaryotic adventures in multicellularity. Current opinion in microbiology. 2003; 6(6): p. 578-585.

25. Watnick P, Kolter R. Biofilm, city of microbes. Journal of bacteriology 2000; 182(10): p. 2675-2679.

26. O’Toole G, Kaplan HB, Kolter R. Biofilm formation as microbial development. Annual Reviews in Microbiology. 2000; 54(1): p. 49-79.

27. Stewart PS, Costerton JW. Antibiotic resistance of bacteria in biofilms. The lancet. 2001; 358(9276): p. 135-138.

28. Donlan RM, Costerton JW. Biofilms: survival mechanisms of clinically relevant microorganisms. Clinical microbiology reviews. 2002; 15(2): p. 167-193.

29. Singh PK, Schaefer AL, Parsek MR, et al. Quorum-sensing signals indicate that cystic fibrosis lungs are infected with bacterial biofilms. Nature. 2000; 407(6805): p. 762-764.

30. Saraswathi P, Beuerman RW. Corneal biofilms: from planktonic to microcolony formation in an experimental keratitis infection with Pseudomonas aeruginosa. The ocular surface. 2015; 13(4): p. 331345 .

31. Williams MM, Yakrus MA, Arduino MJ, et al. Structural analysis of biofilm formation by rapidly and slowly growing nontuberculous mycobacteria. Applied and environmental microbiology. 2009; 75(7): p. 2091-2098.

32. Yam JKH, et al. Cyclic-di-GMP is required for corneal infection by Pseudomonas aeruginosa and modulates host immunity. bioRxiv. 2017.

33. Aung TT, Chor WHJ, Yam JKH, etal. Discovery of novel antimycobacterial drug therapy in biofilm of pathogenic nontuberculous mycobacterial keratitis. The Ocular Surface. 2017.

34. Costerton JW, Stewart SP, Greenberg EP. Bacterial biofilms: a common cause of persistent infections. Science. 1999; 284(5418): p. 13181322.

35. Qin Z, Ou Y, Yang L, et al. Role of autolysin-mediated DNA release in biofilm formation of Staphylococcus epidermidis. Microbiology. 2007; 153(7): p. 2083-2092.

36. Thomas VC, Thurlow LR, Boyle D, et al, Regulation of autolysisdependent extracellular DNA release by Enterococcus faecalis extracellular proteases influences biofilm development. Journal of bacteriology. 2008; 190(16): p. 5690-5698.

37. Allesen-Holm M, Barken KB, Yang L, et al. A characterization of DNA release in Pseudomonas aeruginosa cultures and biofilms. Molecular microbiology. 2006; 59(4): p. 1114-1128.

38. Das T, Manefield M. Pyocyanin promotes extracellular DNA release in Pseudomonas aeruginosa. PloS one. 2012; 7(10): p. e46718.

39. Barnes AM, Ballering KS, Leibman RS, et al. Enterococcus faecalis produces abundant extracellular structures containing DNA in the absence of cell lysis during early biofilm formation. MBio. 2012; 3(4): p. e00193-12.

40. Sahu PK, Iyer PS, Oak AM, et al. Characterization of eDNA from the clinical strain Acinetobacter baumannii AIIMS 7 and its role in biofilm formation. The Scientific World Journal. 2012; 2012.

41. Rose SJ, Bermudez LE. Identification of Bicarbonate as a Trigger and Genes Involved with Extracellular DNA Export in Mycobacterial Biofilms. MBio. 2016; 7(6): p. e01597-16.

42. Whitchurch CB, Tolker-Nielsen T, Ragas PC, et al. Extracellular DNA required for bacterial biofilm formation. Science. 2002; 295(5559): . 1487-1487.

43. Okshevsky M, Meyer RL. The role of extracellular DNA in the establishment, maintenance and perpetuation of bacterial biofilms. Critical reviews in microbiology. 2015.

44. Griffith F. The significance of pneumococcal types. Journal of Hygiene. 1928; 27(02): p. 113-159.

45. Thomas CM, Nielsen KM. Mechanisms of, and barriers to, horizontal gene transfer between bacteria. Nature reviews microbiology. 2005; 3(9): p. 711-721.

46. Mulcahy H, Charron-Mazenod L, Lewenza S. Extracellular DNA chelates cations and induces antibiotic resistance in Pseudomonas aeruginosa biofilms. PLoS Pathog. 2008; 4(11): p. e1000213.

47. Flemming HC, Wingender J. The biofilm matrix. Nature Reviews Microbiology. 2010; 8(9): p. 623-633.

48. Aung TT, Yam JK, Lin S, et al. Biofilms of pathogenic nontuberculous mycobacteria targeted by new therapeutic approaches. Antimicrobial agents and chemotherapy. 2016; 60(1): p. 24-35.

49. Rose SJ, Babrak LM, Bermudez LE. Mycobacterium avium possesses extracellular DNA that contributes to biofilm formation, structural integrity, and tolerance to antibiotics. PloS one. 2015; 10(5): p. e0128772.

50. Ford JG, Huang AJ, Pflugfelder SC, et al. Nontuberculous mycobacterial keratitis in south Florida. Ophthalmology. 1998; 105(9): p. 16521658.

51. Lazar M. Mycobacterium fortuitum keratitis. American journal of ophthalmology. 1974; 78(3): p. 530-532.

52. HU FR. Infectious crystalline keratopathy caused by Mycobacterium fortuitum and Pseudomonas aeruginosa. American journal of ophthalmology. 1990; 109(6): p. 738-739.

53. Shrestha NK, Tuohy MJ, Hall GS, et al. Detection and differentiation of Mycobacterium tuberculosis and nontuberculous mycobacterial isolates by real-time PCR. Journal of clinical microbiology. 2003; 41(11): p. 5121-5126 
54. Palani D, Kulandai LT, Naraharirao MH, et al. Application of polymerase chain reaction-based restriction fragment length polymorphism in typing ocular rapid-growing nontuberculous mycobacterial isolates from three patients with postoperative endophthalmitis. Cornea. 2007; 26(6): p. 729-735.

55. Wunsh SE, Byle GL, Leopold IH, et al. Mycobacterium fortuitum infection of corneal graft. Archives of Ophthalmology. 1969; 82(5): p. 602-607.

56. Abshire R, Cockrum P, Crider J, et al. Topical antibacterial therapy for mycobacterial keratitis: potential for surgical prophylaxis and treatment. Clinical therapeutics. 2004; 26(2): p. 191-196.

57. Aylward G, Stacey A, Marsh R. Mycobacterium chelonei infection of a corneal graft. British journal of ophthalmology. 1987; 71(9): p. 690693.

58. Eiferman RA, Stagner JI. Intraocular penetration of amikacin: iris binding and bioavailability. Archives of Ophthalmology. 1982; 100(11): p. 1817-1819.

59. Alexandrakis G, Alfonso EC, Miller D. Shifting trends in bacterial keratitis in south Florida and emerging resistance to fluoroquinolones. Ophthalmology. 2000; 107(8): p. 1497-1502.

60. Moshirfar M, Meyer JJ, Espandar L. Fourth-generation fluoroquinolone-resistant mycobacterial keratitis after laser in situ keratomileusis. Journal of Cataract \& Refractive Surgery. 2007; 33(11): p. 1978-1981.

61. Chang MA, Jain S, Azar DT. Infections following laser in situ keratomileusis: an integration of the published literature. Survey of ophthalmology. 2004; 49(3): p. 269-280.

62. Fowler AM, Dutton JJ, Fowler WC, et al. Mycobacterium chelonae canaliculitis associated with SmartPlug use. Ophthalmic Plastic \& Reconstructive Surgery. 2008; 24(3): p. 241-243.

63. Wallace R, Bedsole G, Sumter G, et al. Activities of ciprofloxacin and ofloxacin against rapidly growing mycobacteria with demonstration of acquired resistance following single-drug therapy. Antimicrobial agents and chemotherapy. 1990; 34(1): p. 65-70.

64. Chu HS, Hu FR. Non-tuberculous mycobacterial keratitis. Clinical Microbiology and Infection. 2013; 19(3): p. 221-226. 